\title{
Exploratory analysis of social cognition and neurocognition in individuals at clinical high risk for psychosis
}

\author{
Emma Yong ${ }^{a}$, Mariapaola Barbato ${ }^{b}$, David L. Penn ${ }^{c}$, Richard S.E. Keefe ${ }^{d}$, Scott W. Woods ${ }^{e}$, \\ Diana O. Perkins ${ }^{\dagger}$, and Jean Addington ${ }^{*}, \mathrm{~b}$ \\ aDepartment of Psychology, University of Lethbridge, Alberta, Canada \\ bHotchkiss Brain Institute, Department of Psychiatry, University of Calgary, Alberta, Canada \\ 'Department of Psychology, University of North Carolina at Chapel Hill, Chapel Hill NC, United \\ States \\ ${ }^{d}$ Department of Psychiatry, Duke University, Durham, North Carolina, United States \\ eDepartment of Psychiatry, Yale University, New Haven, CT, USA \\ fDepartment of Psychiatry, University of North Carolina at Chapel Hill, Chapel Hill NC, United \\ States
}

\section{Abstract}

\begin{abstract}
Neurocognition and social cognition are separate but related constructs known to be impaired in schizophrenia. The aim of this study was to extend the current knowledge of the relationship between social cognition and neurocognition in individuals at clinical high risk (CHR) of developing psychosis by examining, in a large sample, the associations between a wide range of neurocognitive tasks and social cognition. Participants included 136 young people at CHR. Specific domains within neurocognition and social cognition were compared using Spearman correlations. Results showed that poor theory of mind correlated with low ratings on a wide range of neurocognitive tasks. Facial affect was more often associated with low ratings on spatial working memory and attention. These results supports a link between neurocognition and social cognition even at this early stage of potential psychosis, with indication that poorer performance on social cognition may be associated with deficits in attention and working memory. Understanding these early associations may have implications for early intervention.
\end{abstract}

\section{Keywords}

schizophrenia; theory of mind; facial affect recognition; working memory; attention

\footnotetext{
(C) 2014 Elsevier Ireland Ltd. All rights reserved.

*Corresponding Author: Jean Addington, Mathison Centre for Mental Health Research and Education, University of Calgary, 3280 Hospital Drive NW, Calgary, Alberta, Canada, T2N 4Z6. Tel: 403-210-6379. Fax: 403-210-9182. jmadding@ucalgary.ca.

Publisher's Disclaimer: This is a PDF file of an unedited manuscript that has been accepted for publication. As a service to our customers we are providing this early version of the manuscript. The manuscript will undergo copyediting, typesetting, and review of the resulting proof before it is published in its final citable form. Please note that during the production process errors may be discovered which could affect the content, and all legal disclaimers that apply to the journal pertain.
} 


\section{1.) Introduction}

Individuals with schizophrenia and other psychotic disorders experience deficits in both neurocognition and social cognition which, although related, most likely represent separate constructs (Addington and Piskulic, 2013). Furthermore, significant relationships have been reported between specific neurocognitive and social cognitive domains. For example, poor affect processing and theory of mind have been frequently shown to be associated with deficits in attention, working memory, executive functioning and IQ (Kohler et al, 2000; Brune et al. 2007; Pijnenborg et al., 2009). Recent research has demonstrated that young people who are at clinical high risk (CHR) for psychosis (Addington and Heinssen, 2012) also evidence impaired neurocognition (Giuliano et al., 2010; Fusar-Poli et al., 2012; Barbato et al., 2013) and social cognition (Addington and Piskulic, 2013), although very few studies have examined the association between social cognition and neurocognition in those at CHR. To date, three studies (Chung, et al., 2008; Stanford et al., 2011; Hur et al., in press) have examined associations between theory of mind (ToM) and neurocognition. All three reported significant associations between ToM and IQ and two reported significant associations with executive functioning (Chung, et al., 2008; Hur et al., in press). In these studies verbal memory was inconsistently associated with ToM. A fourth study demonstrated that facial affect labeling was related to executive functioning but not to IQ (Van Rijn et al., 2011). Some of these studies had relatively small samples and assessed only one domain of social cognition, usually ToM.

The purpose of this study is to extend our knowledge of the relationship between social cognition and neurocognition in those at CHR of developing psychosis by examining, in a larger sample, the associations between a wide range of neurocognitive tasks and social cognition. We predict that social cognitive deficits will be positively associated with a range of neurocognitive difficulties, in particular attention, working memory, executive function, and IQ.

\section{2.) Methods}

Data was collected at the initial assessment of PREDICT, a longitudinal study to determine predictors of conversion in individuals at CHR for psychosis, conducted at the Universities of North Carolina (UNC), Toronto, and Yale.

\subsection{Participants}

Participants were 136 CHR individuals (57 Toronto, 54 UNC, 25 Yale). All participants met the Criteria of Prodromal Syndromes (COPS) for the attenuated positive symptom syndrome (APSS), based on the Structured Interview for Prodromal Syndromes (SIPS) (McGlashan et al., 2010), which includes the emergence or worsening of a non-psychotic level of disturbance in thought content, thought process, or perceptual abnormalities over the past year. Exclusion criteria were: presence of an axis I psychotic disorder, history of antipsychotic medications, past or current history of a clinically significant central nervous system disorder, and IQ<70. Furthermore, participants were excluded if they were using antipsychotics at baseline. 


\subsection{Measures}

Criteria for a prodromal syndrome were determined using the SIPS and severity of prodromal symptoms with the Scale of Prodromal Symptoms (SOPS) (McGlashan et al., 2010). The structured clinical interview for DSM-IV (First et al., 1998) was used to assess for the presence of non-psychotic disorders.

Assessment of social cognition included three measures of affect processing, the Facial Emotion Identification Test (FEIT) the Facial Emotion Discrimination Test (FEDT), (Kerr and Neale, 1993) and affective prosody (AP) (Edwards et al., 2001). The Abbreviated Trustworthiness (Adolphs et al., 1998) was used to assess complex social judgments. The Reading the Mind in the Eyes Test (RMET) (Baron-Cohen et al., 2001) was used to assess theory of mind abilities.

In the FEIT participants are presented with facial photographs depicting happiness, sadness, anger, fear, surprise, disgust or neutral and are asked to identify the emotion. In the FEDT, using the same emotions, participants are presented with two different people displaying either the same or different emotions and participants are asked to judge whether the two picture show the same or different emotions. The AP involves audio recordings of four simple sentences (i.e. "he will come soon", "they must stay here", "she will drive fast" and "we must go there") spoken by three professional actors displaying the following emotions: fear, sadness, anger, surprise and neutral resulting in a total of 60 items. Based on sentence recordings participants were required to indicate which emotion was expressed. For each actor, there were three practice and 20 target items, with eight seconds of silence between each item. In the Abbreviated Trustworthiness task, participants are shown 42 faces of unfamiliar people and are asked to imagine they had to trust the pictured person with their money or with their life. They rate how much they would trust the person on a 7-point scale, ranging from -3 (very untrustworthy) to +3 (very trustworthy). The most trustworthy (top third/a score greater than +1 ) and least trustworthy faces (bottom third/a score less than -1) according to Adolphs' normative scores are used to form two scales: the average rating on "trustworthy" faces and the average rating on "untrustworthy" faces. In the RMET participants are shown a pair of eyes and asked to choose among four words the one that best describes what the person is thinking or feeling. The percentage of correct responses is used as a summary score for this measure, consistent with previous research.

Neurocognition was assessed with a comprehensive battery. Attention was assessed with digit span distractibility (Oltmanns and Neale, 1975) and the Continuous Performance Testidentical pairs (Cornblatt and Keilp, 1994); executive functioning with the (Wisconsin Card Sorting Test (Kongs et al., 2000), Trail Making B (Reitan and Wolfson, 1985) and the Stroop Color-Word Test (Golden, 1978); processing speed with the Trail Making Task A (Reitan and Wolfson, 1985); verbal fluency with the Controlled Oral Word Association Test and Category Instances (Benton and Hamsher, 1983); verbal memory with the Rey Auditory Verbal Learning Test (Rey, 1958), verbal working memory with the Letter-Number Sequencing Test (Gold et al., 1970); and spatial working memory with the Computerized Test of Visuospatial Working Memory(Lyons-Warren et al., 2004 and the N-back task (Kirchner, 1958). IQ was assessed with the block design, arithmetic, digit symbol/coding, 
vocabulary, and information subtests of the Wechsler Adult Intelligence Test/Wechsler Intelligence Scale for children-III (Wechsler, 1974, 1981). These tests are presented in Table 1 .

\subsection{Procedures}

Clinical raters were experienced research clinicians who demonstrated adequate reliability at routine checks. Gold standard post-training agreement on the distinction between high risk and psychotic levels of intensity was excellent $(\mathrm{kappa}=0.90)$. Research assistants were trained by DLP for social cognitive assessments and by RK for neurocognitive assessments. Study protocols and informed consents were reviewed and approved by the ethical review boards at all sites.

\subsection{Statistical Analysis}

Two-tailed Spearman correlations were used to determine associations between neurocognitive and social cognitive tasks. In addition, correlations between symptoms and neurocognitive and social cognitive tasks were performed to determine whether prodromal symptoms had to be included in the analysis as a covariate. To account for multiple comparisons, a Bonferroni correction was applied.

\section{3.) Results}

The sample consisted of 136 CHR individuals ( 80 males, 56 females) with a mean age of 20 years ( $\mathrm{SD}=4.7$, range $12-31)$. The majority were single $(94.1 \%)$ and fifty-four percent had completed high school. Eighty percent of our sample was Caucasian, the remaining were African American (7\%), Asian (7\%), native Hawaiian or other Pacific islanders (1\%) and mixed race (5\%). Nineteen percent of the participants had an anxiety disorder and $23.8 \%$ had current depression. For the majority of our participants there was little evidence of alcohol and marijuana abuse (5\% and 14\% respectively). The use of substances other than alcohol and marijuana was either minimal or absent.

The means and standard deviations for the tasks are presented in Table 1 along with means and standard deviations from published norms or healthy control groups published in the literature. Kolmogorov-Smirnov test showed that, with the exception of FEIT, RAVLT, Stroop, CPT d', COWAT and Category instances, our measures were not normally distributed. Therefore we used Spearman correlations. Mean score for the SOPS positive symptoms was 10.88 ( $\mathrm{SD}=3.04)$ and for SOPS negative symptoms the mean was 8.04 $(\mathrm{SD}=5.78)$ There were no significant associations between positive and negative symptoms as assessed by the SOPS and any of the neurocognitive or social cognitive tasks. Thus, we did not control for symptoms in the analyses. Associations between social cognition and neurocognition are presented in Table 2 . The trustworthiness task was unrelated to any of the neurocognitive tasks. IQ was unrelated to social cognition. Low ratings on the AP and RMET correlated with low ratings on almost all neurocognitive tasks, but only correlations with RMET remained significant after the Bonferroni correction. Low ratings on FEIT was also associated with low ratings on most of the neurocognitive tests, but only remained 
significant with N-back tasks 1 and 2 and the CPT-IP (composite score) after applying a Bonferroni correction.

\section{4.) Discussion}

This study examined associations between individual neurocognitive tasks and social cognition in individuals at CHR for psychosis. It was observed in this sample that IQ was unrelated to social cognition. This is contrary to previous findings with CHR samples in the literature (Chung et al., 2008; van Rijn et al 2011; Hur et al., 2013). However it has to be noted that the mean IQ in our sample (IQ=112.06) was slightly higher than that of other at risk samples respectively from the above studies (109.76, 109.47, and 99.9). The trustworthiness task was unrelated to neurocognition, a result that has been observed with other patient populations (Bellugi et al., 1999). Since judgments of trustworthiness are typically made rapidly and they have been suggested to result from over-learned automatic processes (Haut and McDonald, 2010), it is possible that they are independent from neurocognition.

Poorer performance on the RMET was significantly and positively associated with deficits on a wide range of cognitive tasks, mainly involving attention and memory. Similarly, facial affect identification was also positively associated with attention and working memory, partially confirming what has been observed in schizophrenia patients (e.g. Kohler et al., 2000). Affect discrimination was unrelated to cognition, possibly because an examination of their scores compared to norms from the literature suggests that as a group, participants were not impaired on FEDT with very little variation. There was no relationship between symptoms and social cognition, which is consistent with previous reports (Stanford et al., 2011), although a link between symptom progression and social cognition has been reported (Kim et al., 2011).

In this study we had a large sample of CHR participants and no confound of antipsychotic use. However, the study was limited in that we did not have a healthy control group, which would have allowed us to determine more specifically if social cognition and neurocognition were impaired in our sample. An examination of the mean scores from published norms or healthy controls in other studies suggest that our sample is most likely impaired on many of the cognitive tasks, as would be expected from the literature. Although, our sample typically had lower ratings on most of the social cognitive tasks but without statistical comparison with a healthy control group it is not possible to determine if these differences are significant.

In conclusion, our data supports a link between neurocognition and social cognition, and suggests that poorer performance on social cognition may be associated to deficits in neurocognition, in particular attention and working memory, as well as verbal memory and executive functioning. This is consistent with a recent meta-analysis examining social cognition and neurocognition in both first episode patients and those with a more chronic course of illness (Ventura et al. 2013). This study reported that no one domain or group of neurocognitive domains (based on the MATRICS) was obviously more strongly related to any of the domains of social cognition. Relevant for this current study were the moderate 
and consistent relationships that were observed in these samples of individuals with schizophrenia amongst theory of mind, perception and emotion perception with verbal, visual and working memory, attention and reasoning. Ventura et al. (2013) reported that the relative strength of the relationships was medium with aggregated $r$ 's ranging from $r=0.23$ to $r=0.34$. In our study, once the bonferroni correction was applied r's ranged from 0.36 to 0.46. This suggests that our data fits with what is being reported in the literature with respect to individuals with full blown psychotic illnesses.

An improved understanding of the role of social cognition and neurocognition in this at risk period may contribute to a greater understanding of how these deficits that are notable in schizophrenia develop and eventually their role, if any, in the later development of psychosis. Increased information about deficits in social cognition does have implications for potential treatments that have had some success in schizophrenia (Kurtz and Richardson, 2012). Such treatments may be particularly valuable for individuals at CHR, who present only early signs of psychosis and have a higher recovery potential due to ongoing neurodevelopment during adolescence and young adulthood (Statucka \& Walder, 2013).

\section{Acknowledgments}

This study was supported by an Alberta Innovates-Health Solutions summer studentship to Emma Yong and NIMH grants U01U01MH06634 to J. Addington, U01 MH066069-04 to D. Perkins, U01MH066160 to S. Woods.

\section{References}

Addington J, Heinssen R. Prediction and prevention of psychosis in youth at clinical high risk. Annual Review of Clinical Psychology. 2012; 8:269-289.

Addington, J.; Piskulic, D. Social cognition early in the course of the illness. In: Roberts, D.; Penn, D., editors. Social Cognition in Schizophrenia. Oxford University Press; New York: 2013. p. 245-262.

Addington J, Piskulic D, Perkins D, Woods SW, Liu L, Penn DL. Affect recognition in people at clinical high risk of psychosis. Schizophrenia Research. 2012; 140:87-92. [PubMed: 22763425]

Adolphs R, Tranel D, Damasio AR. The human amygdala in social judgment. Nature. 1998; 393:470474. [PubMed: 9624002]

Barbato M, Colijn MA, Keefe RS, Perkins DO, Woods SW, Hawkins KA, Christensen BK1, Addington J. The course of cognitive functioning over six months in individuals at clinical high risk for psychosis. Psychiatry Research. 2013; 206:195-199. [PubMed: 23159196]

Baron-Cohen S, Wheelwright S, Hill J, Raste Y, Plumb I. The "reading the mind in the eyes" test revised version: A study with normal adults and adults with Asperger Syndrome or high functioning autism. Journal of Child Psychology and Psychiatry. 2001; 42:241-251. [PubMed: 11280420]

Bellugi U, Adolphs R, Cassady C, Chiles M. Towards the neural basis for hypersociability in a genetic syndrome. NeuroReport. 1999; 10:1653-1657. [PubMed: 10501552]

Benton, AL.; Hamsher, K. Multilingual Aphasia Examination. 2. AJA Associates; Iowa City: 1983.

Brune M, Abdel-Hamid M, Lehmkamper C, Sonntag C. Mental state attribution, neurocognitive functioning, and psychopathology: what predicts poor social competence in schizophrenia best? Schizophrenia research. 2004; 92:151-159. [PubMed: 17346931]

Chung YS, Kang DH, Shin NY, Yoo SY, Kwon JS. Deficit of theory of mind in individuals at ultrahigh-risk for schizophrenia. Schizophrenia Research. 2008; 99:111-118. [PubMed: 18096371]

Cornblatt B, Keilp J. Impaired attention genetics, and the pathophysiology of schizophrenia. Schizophrenia Bulletin. 1994; 20:31-46. [PubMed: 8197420]

Couture SM, Penn DL, Addington J, Woods SW, Perkins DO. Assessment of social judgments and complex mental states in the early phases of psychosis. Schizophrenia Research. 2008; 100:237241. [PubMed: 18255273] 
Edwards J, Pattison PE, Jackson HJ, Wales RJ. Facial affect and affective prosody recognition in firstepisode schizophrenia. Schizophrenia Research. 2001; 48:235-53. [PubMed: 11295377]

First, MB.; Spitzer, RL.; Gibbon, M.; Williams, JBW. Structured clinical interview for DSM-IV patient edition (SCID-I/P V and SCID-I/NP Version 2.0). New York: Biometric Research Department; 1998.

Fusar-Poli P, Deste G, Smieskova R, Barlati S, Yung AR, Howes O, Stieglitz RD, Vita A, McGuire P, Borgwardt S. Cognitive functioning in prodromal psychosis: A meta-analysis. Archives of General Psychiatry. 2012; 69:562-571. [PubMed: 22664547]

Giuliano AJ, Li H, Mesholam-Gately RI, Sorenson S, Woodberry KA, Seidman LJ. Neurocognition in the psychosis risk syndrome: a quantitative and qualitative review. Current Pharmaceutical Design. 2012; 18:399-415. [PubMed: 22239571]

Gold JM, Carpenter C, Randolph C, Goldberg TE, Weinberger DR. Auditory working memory and Wisconsin Card Sorting Test performance in schizophrenia. Archives of General Psychiatry. 1997; 54:159-165. [PubMed: 9040284]

Golden, C. Stroop Color and Word Test Manual. Stoelting; Chicago: 1978.

Goudsmit N, Coleman E, Seckinger RA, Wolitzky R, Stanford AD, Corcoran C, Goetz RR, Malaspina D. A brief smell identification test discriminates between deficit and non-deficit schizophrenia. Psychiatry Research. 2003; 120:155-164. [PubMed: 14527647]

Haut KM, MacDonald AW 3rd. Persecutory delusions and the perception of trustworthiness in unfamiliar faces in schizophrenia. Psychiatry Research. 2010; 178 (3):456-460. [PubMed: 20569994]

Hur JW, Byun MS, Shin NY, Shin YS, Kim SN, Jang JH, Kwon JS. General intellectual functioning as a buffer against theory-of-mind deficits in individuals at ultra-high risk for psychosis. Schizophrenia Research. 2013; 149:83-87. [PubMed: 23810120]

Kahn PV, Walker TM, Williams TS, Cornblatt BA, Mohs RC, Keefe RS. Standardizing the use of the Continuous Performance Test in schizophrenia research: a validation study. Schizophrenia Research. 2012; 142:153-158. [PubMed: 23040735]

Kerr SL, Neale JM. Emotion perception in schizophrenia: specific deficit or further evidence of generalized poor performance? Journal of Abnormal Psychology. 1993; 102:312-318. [PubMed: 8315144]

Kim HS, Shin NY, Jang JH, Kim E, Shim G, Park HY, Hong KS, Kwon JS. Social cognition and neurocognition as predictors of conversion to psychosis in individuals at ultra- high risk. Schizophrenia Research. 2011; 130:170-175. [PubMed: 21620681]

Kirchner W. Age differences in short-term retention of rapidly changing information. Journal of Experimental Psychology. 1958; 55:352-358. [PubMed: 13539317]

Kohler CG, Bilker W, Hagendoorn M, Gur RE, Gur RC. Emotion recognition deficit in schizophrenia: association with symptomatology and cognition. Biological psychiatry. 2000; 48:127-136. [PubMed: 10903409]

Kongs, LL.; Thompson, GL.; Iverson, GL.; Heaton, RK. Wisconsin Card Sorting Test-64 Card Version: Professional Manual Psychological Assessment Resources. Odessa: 2000.

Kurtz MM, Richardson CL. Social Cognitive Training for Schizophrenia: A Meta-Analytic Investigation of Controlled Research. Schizophrenia Bulletin. 2012; 38 (5):1092-1104. [PubMed: 21525166]

Lyons-Warren A, Lillie R, Hershey T. Short- and long-term spatial delayed response performance across the lifespan. Developmental Neuropsychology. 2004; 26:661-678. [PubMed: 15525563]

McGlashan, TH.; Walsh, BC.; Woods, SW. The Psychosis-Risk Syndrome: Handbook for Diagnosis and Follow-Up. Oxford University Press; New York: 2010.

Miller KM, Price CC, Okun MS, Montijo H, Bowers D. Is the n-back task a valid neuropsychological measure for assessing working memory? Archives of Clinical Neuropsychology. 2009; 24:711717. [PubMed: 19767297]

Oltmanns TF, Neale JM. Schizophrenic performance when distractors are present: attentional deficit or differential task difficulty? Journal of Abnormal Psychology. 1975; 84:205-209. [PubMed: 1133249] 
Penn DL, Combs DR, Ritchie M, Francis J, Cassisi J, Morris S, Townsend M. Emotion recognition in schizophrenia: further investigation of generalized versus specific deficit models. Journal of Abnormal Psychology. 2000; 109:512-516. [PubMed: 11016120]

Pijnenborg GH, Withaar FK, Evans JJ, van den Bosch RJ, Timmerman ME, Brouwer WH. The predictive value of measures of social cognition for community functioning in schizophrenia: implications for neuropsychological assessment. Journal of the International Neuropsychological Society. 2009; 15:239-247. [PubMed: 19203437]

Pinkham AE, Penn DL. Neurocognitive and social cognitive predictors of interpersonal skill in schizophrenia. Psychiatry Research. 2006; 143:167-178. [PubMed: 16859754]

Reitan, R.; Wolfson, D. The Halstead-Reitan Neuropsychological Test Battery. Neuropsychology Press; Tucson: 1985.

Rey, A. The Clinical Examination in Psychology. Presse Universitaire de France; Paris: 1958.

Scholes KE, Martin-Iverson MT. Cannabis use and neuropsychological performance in healthy individuals and patients with schizophrenia. Psychological Medicine. 2010; 40:1635-1645. [PubMed: 20018124]

Stanford AD, Messinger J, Malaspina D, Corcoran CM. Theory of mind in patients at clinical high risk for psychosis. Schizophrenia Research. 2011; 131:11-17. [PubMed: 21757324]

Statucka M, Walder DJ. Efficacy of social cognition remediation targeting facial affect recognition in schizophrenia: a systematic review with consideration of high-risk samples and sex differences. Psychiatry Research. 2013; 206:125-319. [PubMed: 23375627]

Strauss, E.; Sherman, E.; Spreen, O. A Compendium of Neuropsychological Tests: Administration, Norms, and Commentary. Oxford University Press; New York: 2006. p. 1042-1052.

Tombaugh TN. Trail Making Test A and B: normative data stratified by age and education. Archives of Clinical Neuropsychology. 2004; 19:203-214. [PubMed: 15010086]

van Rijn S, Aleman A, de Sonneville L, Sprong M, Ziermans T, Schothorst P, van Engeland H, Swaab H. Misattribution of facial expressions of emotion in adolescents at increased risk of psychosis: The role of inhibitory control. Psychological Medicine. 2011; 41:499-508. [PubMed: 20507669]

Wechsler, D. Manual for the Wechsler Intelligence Scale for Children-Revised. Psychological Corporation; New York: 1974.

Wechsler, D. Manual for the Wechsler Adult Intelligence Scale-Revised. Psychological Corporation; New York: 1981. 


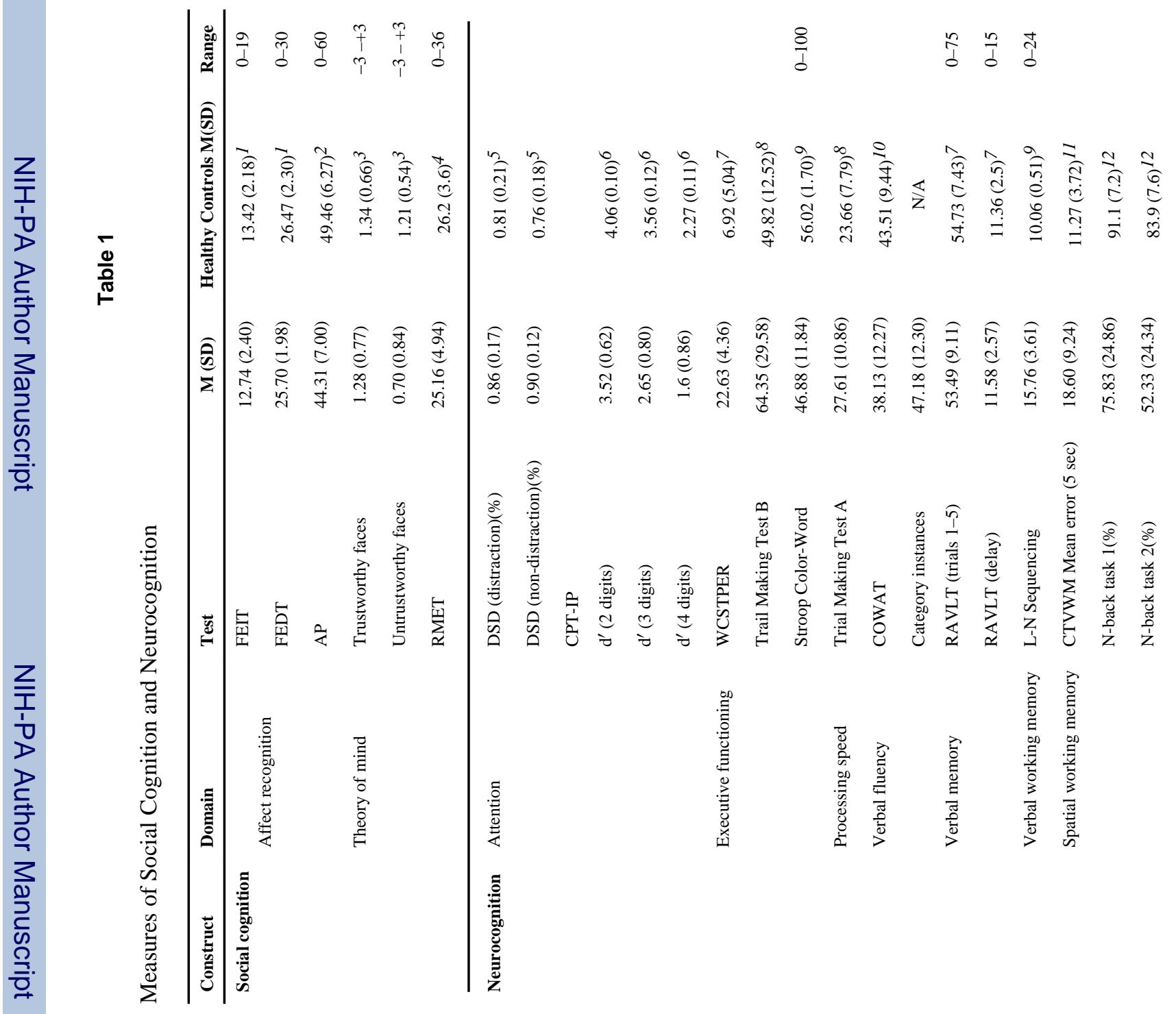




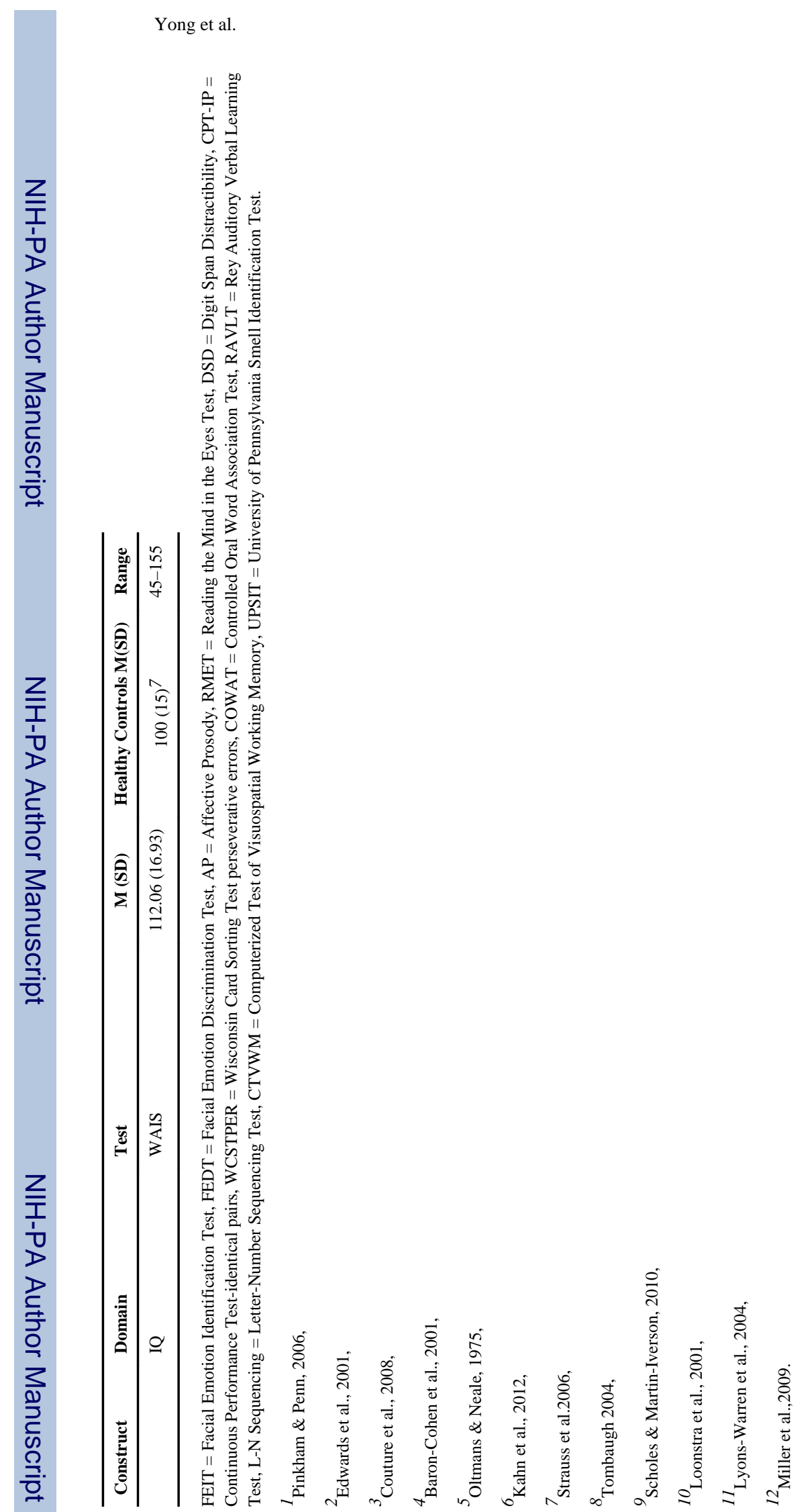

Psychiatry Res. Author manuscript; available in PMC 2015 August 15. 


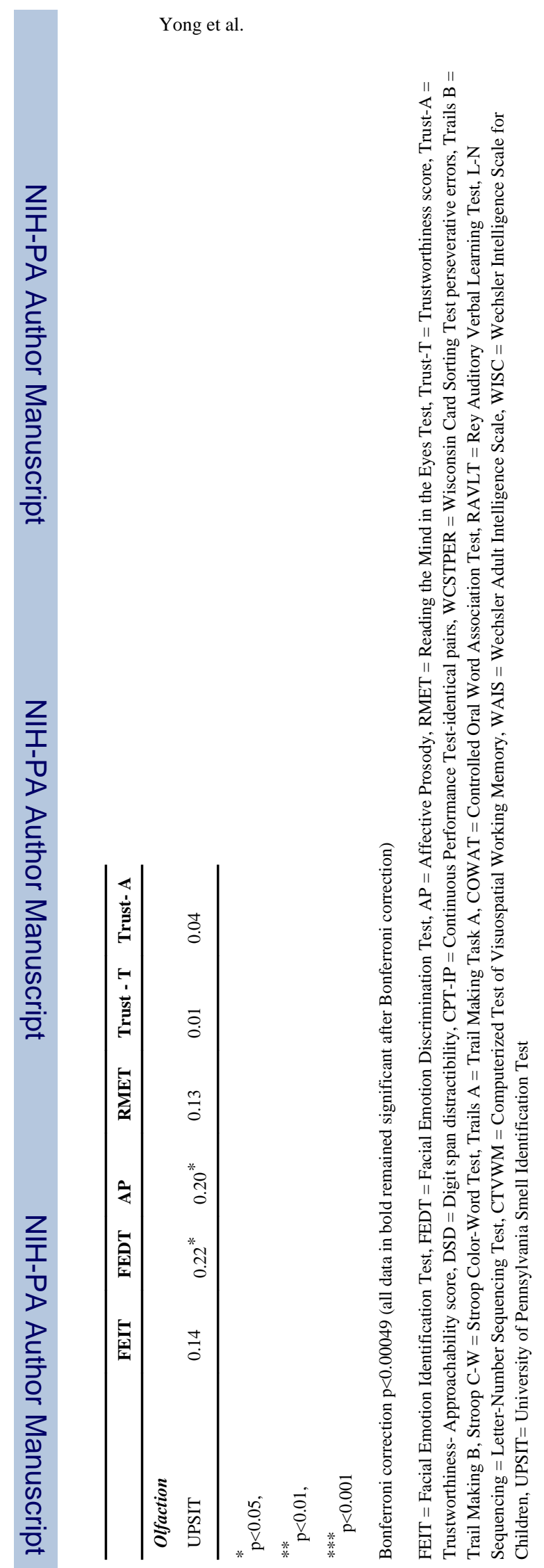

Psychiatry Res. Author manuscript; available in PMC 2015 August 15. 